\title{
Emergence and outbreak of carbapenemase-producing KPC-3 Klebsiella pneumoniae in Spain, September 2009 to February 2010: control measures
}

A Robustillo Rodela1 ${ }^{1}$ C Díaz-Agero Pérez ${ }^{1}$, T Sanchez Sagrado ${ }^{1}$, P Ruiz-Garbajosa², M J Pita López ${ }^{1}$, V Monge (vmonge.hrc@salud. madrid.org) ${ }^{1}$

1. Service of Preventive Medicine, University Hospital Ramón y Cajal, and Health Research Institute (Instituto Ramón y Cajal de Investigación Sanitaria; IRYCIS), Madrid, Spain

2. Microbiology Service, University Hospital Ramón y Cajal, Biomedical Research Centre Network for Epidemiology and Public Health (CIBER en Epidemiología y Salud Pública) and Health Research Institute (Instituto Ramón y Cajal de Investigación Sanitaria; IRYCIS), Madrid, Spain

Robustillo Rodela A, Díaz-Agero Pérez C, Sanchez Sagrado T, Ruiz-Garbajosa P, Pita López MJ, Monge V. Emergence and outbreak of carbapenemase-producing KPC-3 Klebsiella pneumoniae in Spain, September 2009 to February 2010: control measures.

Euro Surveill. 2012;17(7):pii=20086. Available online: http://www.eurosurveillance.org/ViewArticle.aspx?Articleld=20086

Article published on 16 February 2012

This report describes the epidemiological features of the first outbreak caused by $\mathrm{KPC}_{3}$ carbapenemase-producing Klebsiella pneumoniae (KPC-3-KP) in Spain and how it was effectively controlled. From 16 September 2009 to the end of February 2010, seven patients infected or colonised with KPC-3-KP were detected. Stool surveillance cultures were recovered from patients, doctors, nurses, nursing assistants, cleaners and hospital porters working in the affected units. Hand swabs were taken from workers and patients' relatives for culturing. Environmental samples were also taken. Patients infected or colonised with KPC-3-KP were placed in single rooms under contact precautions and $4 \%$ chlorhexidine soap was used for their daily hygiene. Staff attended educational seminars and workshops on hand hygiene and isolation of patients. An alcohol-based disinfectant was used for surface cleaning and disinfecting. The floor was cleaned with a disinfectant containing benzalkonium chloride and didecyldimethylammonium. All samples collected were negative for KPC-3-KP. After implementing the control measures, no further cases were reported in the affected units. All cases had comorbidities, long hospital stay and aggressive/intensive antimicrobial treatment. This study emphasises the importance of early intensification of infection control to interrupt the transmission of KPC-producing organisms.

\section{Introduction}

Carbapenems are widely regarded as the drugs of choice for the treatment of severe infections caused by extended-spectrum beta-lactamase (ESBL)-producing Enterobacteriaceae [1]. The emergence of carbapenemresistant enterobacteria is worrisome, since antimicrobial treatment options are very restricted [2].
Carbapenemases are a large and diverse family of microbial enzymes that hydrolyse not only carbapenems but also other beta-lactam antibiotics. One of the most important groups is the KPC-enzymes, classified as beta-lactamases Ambler class A and Bush functional group 2, that hydrolyse all beta-lactams except cephamycins [3].

KPC-producing Klebsiella pneumoniae were first isolated in North Carolina in 1996 [4] and until 2004 these enzymes were found only in the United States [5-7]. The first outbreak of KPC-producing $K$. pneumoniae outside the United States was described in Tel Aviv in 2006 [8]. The strains isolated in Israel were genetically identical to the ones previously isolated in the United States. This supported the hypothesis that the KPC producer was transferred from the United States to Israel [9]. The European country in which most cases have been reported so far is Greece, where the situation can be described as endemic [10-15]. Italy and France have recently described a rapid increase in the number of of cases $[16,17]$.

We report here the epidemiological features of the first outbreak by $\mathrm{KPC}_{3}$ carbapenemase-producing $\mathrm{K}$. pneumoniae (KPC-3-KP) in Spain and how it was controlled.

\section{Material and methods \\ Outbreak investigation}

Ramon y Cajal Hospital is a 1,090-bed university teaching hospital located in Madrid, Spain. From 16 September 2009 through the end of February 2010, seven patients with infection or colonisation with KPC-3-KP were detected. Four of these patients were admitted to two different but adjacent units: internal medicine and oncology. Both units share hospital porters and cleaning staff and some medical equipment 
such as electrocardiographs. Of the remaining three patients, one was detected in the paediatric unit on a different floor during the same period, and the other two appeared after hospital discharge.

\section{Case patient definition}

Any inpatient infected or colonised with KPC-3-KP was considered as a case. An infected patient was defined as a person with a positive culture for KPC-3-KP who met the Centres for Disease Control and Prevention (CDC) clinical criteria of infection [18]. A patient was defined as colonised, when KPC-3-KP was isolated from surveillance cultures or clinical specimens in the absence of clinical signs of infection.

\section{Clinical data, surveillance, and}

environmental cultures

Clinical records from inpatients were reviewed and the following data were registered: age, sex, diagnosis at the time of hospital admission, comorbid conditions (diabetes mellitus, cardiovascular disease, pulmonary disease, hepatic disease, central nervous system disease, malignancy, anaemia), previous hospital admissions, admission to intensive care unit, treatment with immunosuppressors, antibiotic treatment, invasive procedures (insertion of central venous catheter, insertion of urinary catheter, surgery, mechanical ventilation), antimicrobial resistance pattern, and outcome (recovery/death).

According to infection control and prevention policy in our institution, when a patient has present or previous history of infection/colonisation with a multidrugresistant microorganism or has shared a room with an infected/colonised patient, rectal and pharyngeal swabs are taken to detect colonisation and to decide when to discontinue contact precautions.

During the outbreak, stool surveillance cultures were recovered from patients, doctors, nurses, nursing assistants, cleaners and hospital porters working in the internal medicine and oncology units [19]. Hand surface swabs were taken from workers and patients' relatives and cultured. Subjects placed their fingertips on cystine lactose electrolyte deficient (CLED) agar plates using the four fingers first, followed by the thumb in the middle of the plate. An imprint method was used because it is easy and feasible in our institution. Moreover, with this method we estimated bacteria present on the palm of the hand, which is the anatomical location with the highest risk of transmission. Samples were also taken from working surfaces, taps, patients' rooms (bedrails, sinks, taps and bedside tables), nurses' sinks, computer key boards, pulse oximeters, and sphygmomanometers. Stool and environmental samples were directly inoculated onto MacConkey agar plates supplemented with ceftazidime ( $4 \mathrm{mg} / \mathrm{L}$ ) and using previous broth enrichment (BHI) supplemented with imipenem $(1 \mathrm{mg} / \mathrm{L})$.
Bacterial identification, susceptibility testing, screening for carbapenemase production and molecular laboratory techniques were done as described by Curiao et al. [20].

\section{Outbreak control measures}

Patients were placed in single rooms under contact precautions according to our facility's protocol. A $4 \%$ chlorhexidine soap was used for their daily hygiene. Staff working in the affected units received educational seminars and attended workshops on special hand hygiene and patient isolation. The units were cleaned thoroughly with two products that were also used for the daily cleaning during the whole outbreak period. An alcohol-based disinfectant was used for surface cleaning and disinfecting (Incidin Liquid), and the floor was cleaned with a disinfectant containing benzalkonium chloride and didecyldimethylammonium (Incidin Rapid).

\section{Results}

The outbreak described here involved seven patients. The index case (Case 1) was a patient in their 60 s who had no history of previous hospitalisations. Three months after admission to the oncology unit (third floor, section A), KPC-3-KP was isolated in a urine culture. The patient was discharged five days later.

Two further cases were detected in the adjacent internal medicine unit (third floor, section B) 12 and 37 days after isolation of Case 1 (see Figure). Neither case had a history of previous admission to our hospital. Case 2, a patient in their 50s, had a history of rectal colonisation with ESBL-producing Escherichia coli. Ten days after admission KPC-3-KP was isolated in a rectal culture during a routine investigation of rectal carriage of ESBL-producing isolates. Case 3 had symptoms of urinary tract infection and the urine culture was positive for KPC-3-KP. ESBL-producing Klebsiella oxytoca and $E$. coli were isolated in rectal swabs as well. This patient shared the same room with Case 2 for four days one month before KPC-3-KP isolation.

Case 4 was a teenager admitted to the paediatric unit, (located on the tenth floor). KPC-3-KP was isolated in a wound culture. This patient had previous hospitalisations in our institution, the last one a two-month stay from which they had been discharged only ten days before. This patient had never been admitted to the oncology or the internal medicine unit, and the paediatric unit does not share staff or medical equipment with those units.

Two months after the first case, KPC-3-KP was isolated in a rectal culture from a patient in their 6os (Case 5) who was staying for one month in the oncology unit, at the same time as Case 1, but they did not share the same room at any time. At the time screening for KPC3-KP was performed in the hospital, Case 5 had already been discharged. It was during his next admission, two months after Case 1, that control surveillance cultures 
were grown and $\mathrm{KPC}-3-\mathrm{KP}$ was isolated in a rectal culture. No further cases have been detected since, neither in the oncology unit nor in the internal medicine unit.

Two new cases were reported two months after Case 5. From Case 6 KPC-3KP was isolated in blood and urine cultures taken in the emergency unit. This case was a person in their late $80 \mathrm{~s}$ and had been an inpatient for 10 days during the outbreak period but had not stayed in the affected units. The last case detected (Case 7) a person in their $70 \mathrm{~s}$ previous history of multiple hospitalisations in our institution, was admitted on January 2010. During her stay KPC-3-KP was isolated in a sputum culture. She had been referred to the gastroenterology unit during the outbreak period and she had no known epidemiological association with the rest of the cases. The sequence of case detection is shown in the Figure.

All patients were diagnosed between 10 and 61 days after admission to hospital. All of them had multiple underlying conditions (anaemia: $n=5$; hypertension $n=4$; cancer: $n=3$; diabetes mellitus: $n=1$; Crohn disease: $n=1$; chronic renal insufficiency: $n=1$ ). Five of them were on immunosuppressive treatment. All cases received antibiotic therapy in the month prior the diagnosis: three with amoxicilin/clavulanic, three with meropenem, two with teicoplanin and one with vancomycin.

\section{Microbiological results}

All these isolates were carbapenemase-producing KPC 3. They were considered clonally related and were assigned to the new sequence type ST384. All the isolates were susceptible to amikacin, gentamicin, ciprofloxacin and trimethroprim/sulfamethoxazole and resistant to imipenem, meropenem, cefepime, cefotaxime and piperacillin/tazobactam.

Stool samples were collected from 25 of the other 32 inpatients in the oncology and internal medicine units and from 27 of 39 staff members. All of them were negative for KPC-3-KP.

The 13 hand cultures from health staff were also negative for KPC-3-KP, and the microorganisms isolated were all common skin contaminants. ESBL-producing $K$. pneumoniae was isolated in only one healthcare worker. All five hand samples taken from the patients'

\section{FIGURE}

Admission period and hospital unit of patients with KPC3-producing Klebsiella pneumoniae, Madrid, Spain, 16 September 2009-February $2010(n=7)$

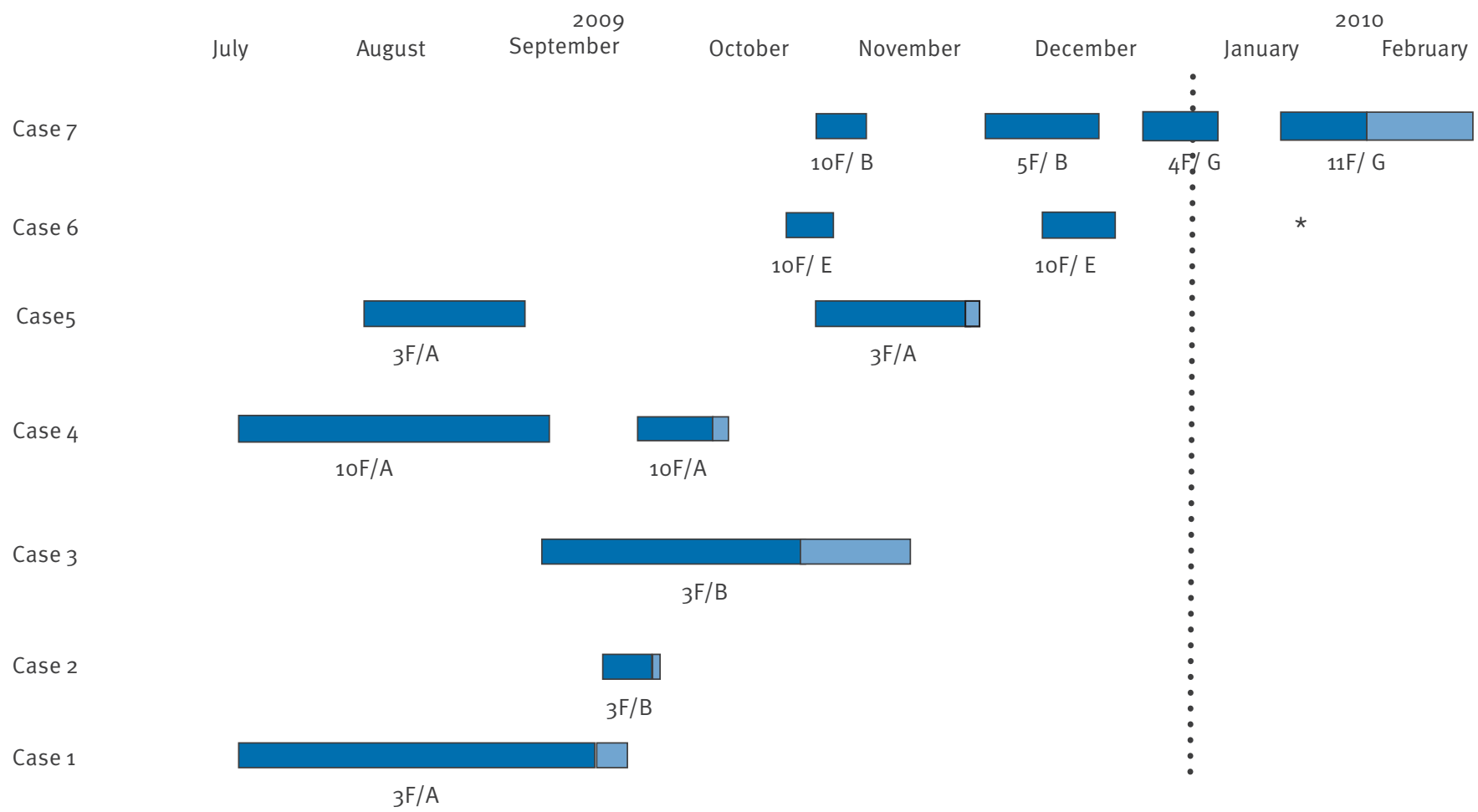

F/_: Floor/Section

Period of admission not infected/colonised

$\square$ Period of admission infected/colonised

* Culture taken in the Emergency Unit 
relatives were negative for KPC-3-KP. The 13 environmental samples were also negative for KPC-3-KP, but positive for other microorganisms as listed in the Table.

\section{Discussion}

We describe the first cases of infection/colonisation with KPC-3 carbapenemase-producing $K$. pneumoniae in Spain. Microbiological data, PCR analysis and molecular techniques showed that all isolates were genetically identical, supporting the hypothesis for a clonal KPC-3-related infectious outbreak [20]. Emergence of $\mathrm{KPC}$-producing $K$. pneumoniae is a significant public health concern.

Outbreaks have been reported in several countries $[7,8,13,21-23]$. In some of those outbreaks, the index case had been previously admitted to a hospital in an endemic area [22,23]. In our study, none of the patients had a previous history of travelling to endemic areas. KPC-3-KP may have been introduced to our facility by an undetected infected or colonised patient, or the index case may have been previously colonised before the KPC-3-KP isolation.

Infection control measures were intensified on the oncology, internal medicine and paediatric wards, where the first five cases were detected. Cases 6 and 7 had been admitted to the hospital during the outbreak period in October 2009, but not to any of the affected units, so no samples had been taken. They were identified as colonised/infected by the same clone only during a later hospital admission in early 2010. Other authors have reported outbreaks in which associated cases were detected from two weeks to five months after the end of the defined outbreak period [21]. We do not know if Cases 6 and 7 were colonised during their earlier hospitalisation in October 2009, since no screening was performed at the time, or if they may have been colonised before admission to the hospital (January-February 2010), since the prevalence of $\mathrm{KPC}$-producing $K$. pneumoniae in the community is unknown.
Studies from Israel and the United States have identified risks factors for nosocomial acquisition of KPC-producing K. pneumoniae. They included poor functional status, ICU stay, transplantation, mechanical ventilation, prolonged hospital stay and antimicrobial treatment [24-26]. Another study that took place in Puerto Rico [21] described further risk factors: wounds, previous surgery and transfer between hospital units. We cannot extract conclusions related to risks factors for KPC-3-K from our study. All the cases detected in our facility had similar characteristics to the ones affected in prior published outbreaks: comorbidities, long hospital stay $[7,13,23]$ and aggressive/intensive antimicrobial treatments $[5,13,15,21]$. In our case, two patients were in an ICU with mechanical ventilation and four underwent surgical procedures.

Antimicrobial pressure may have been a selective factor for the primary colonisation. Use of extended spectrum carbapenems, fluorquinolones and cephalosporins has been identified as a risk factor for carbapenem-resistant $K$. pneumoniae [24-26]. None of our patients had received quinolones one month prior to the infection and all patients had received beta-lactams, as did the patients of the outbreaks described by Purnaras et al. [14] and by Nadkarn [27]. Further studies are needed to clarify the role of different antimicrobials in carbapenem-resistance acquired by $K$. pneumoniae. It may be plausible that the number of antibiotics administered to case patients increases the risk of acquisition of carbapenem-resistance in K. pneumoniae, rather than the administration of a specific antibiotic group [21].

Mortality data described in different papers range from $25 \%$ to $69 \%[7,13-15,21,26]$. In our case series one death was reported. The only patient with bacteraemia by KPC-3-KP died shortly after hospital discharge, and the cause of death is unknown to us. The rest of the patients were successfully treated according to the susceptibility pattern.

\section{TABLE}

Environmental cultures, KPC3-producing Klebsiella pneumoniae hospital outbreak, Madrid, Spain, 16 September 2009February $2010(\mathrm{n}=13)$

\section{Microorganism}

Staphylococcus epidermidis $(\mathrm{n}=8)$

Non-fermenting Gram-negative bacilli $(n=1)$

Streptococcus $s p$. $(\mathrm{n}=1)$

ESBL-producing $K$. pneumoniae $(\mathrm{n}=1)$

K. pneumoniae $(\mathrm{n}=2)$

Bacillus sp. $(\mathrm{n}=1)$

Pseudomonas aeruginosa $(\mathrm{n}=2)$

Enterobacter $s p$. $(\mathrm{n}=1)$
Surface

Bedside tables, bed rails, sinks, sphygmomanometer, working surfaces, computer keyboard Working surface

Working surface

Bedside table

Working surfaces, computer keyboard

Computer keyboard

Taps

Working surfaces

KPC-3-KP: KPC3 carbapenemase-producing K. pneumoniae

a $>1$ microorganism can be isolated in the same sample. 
K. pneumoniae is a common cause of nosocomial pneumonia. Its principal nosocomial reservoirs are contaminated medical equipment, hands of hospital staff and the gastrointestinal tract of patients [9]. In our study, the emergence of a monoclonal outbreak and the impossibility of identifying a common source in an environmental reservoir suggest transmission from patient to patient through the hands of hospital staff, as has been previously described $[23,28,29]$.

Some papers have reported the isolation of KPCproducing $K$. pneumoniae from surfaces, intravenous poles, blood pressure cuffs or endotracheal tube connectors $[5,15]$, while other authors, as well as our own outbreak investigation, did not isolate KPC-3-KP in environmental samples $[13,30]$. However, after thorough cleaning and disinfection, the outbreak did not spread further. We may not have taken enough environmental samples.

Previous studies have shown that KPC-producing $K$. pneumoniae outbreaks are difficult to manage. Monoclonal outbreaks may evolve to polyclonal endemicity if a nosocomial pathogen is not controlled soon after its emergence in a hospital $[14,21]$. Our infection control measures, including contact isolation, were enhanced the moment the first case was detected, and they controlled the outbreak effectively. It is difficult to evaluate the effectiveness of each control measure (chlorhexidine soap, contact isolation, staff training, surface cleaning and disinfection with new products) since all of them were established immediately. The rapid simultaneous application of several measures may have contributed to the effectiveness. We assume that early communication to the staff of the affected units, educational talks and hand hygiene were key factors in controlling the outbreak.

The dissemination of carbapenem-resistant $K$. pneumoniae and the difficulty to treat infections produced by these bacteria with the currently available antimicrobial drugs make it necessary to focus our efforts on early detection and implementation of infection control measures to limit the emergence and transmission of KPC-producing organisms. This issue will require further investigation directed at characterising the molecular mechanisms and selection pressures which promote the spread of these microorganisms. Judicious antimicrobial use should be emphasised.

\section{Acknowledgments}

Molecular studies on KPC isolates were funded by the European Commission (Research grant HEALTH-F3-2008-223031).

\section{References}

1. Pitout JD, Laupland KB. Extended-spectrum beta-lactamaseproducing Enterobacteriaceae: an emerging public-health concern. Lancet Infect Dis. 2008;8(3):159-66.
2. Nordmann P, Cuzon G, Naas T. The real threat of Klebsiella pneumoniae carbapenemase-producing bacteria. Lancet Infect Dis. 2009;9(4):228-36.

3. Munoz-Price LS, Quinn JP. The spread of Klebsiella pneumoniae carbapenemases: a tale of strains, plasmids, and transposons. Clin Infect Dis. 2009;49(11):1739-41.

4. Yigit H, Queenan AM, Anderson GJ, Domenech-Sanchez A, Biddle JW, Steward CD, et al. Novel carbapenem-hydrolyzing beta-lactamase, KPC-1, from a carbapenem-resistant strain of Klebsiella pneumoniae. Antimicrob Agents Chemother. 2001;45(4):1151-61.

5. Bratu S, Landman D, Haag R, Recco R, Eramo A, Alam M, et al. Rapid spread of carbapenem-resistant Klebsiella pneumoniae in New York City: a new threat to our antibiotic armamentarium. Arch Intern Med. 2005;165(12):1430-5.

6. Bratu S, Mooty M, Nichani S, Landman D, Gullans C, Pettinato $B$, et al. Emergence of KPC-possessing Klebsiella pneumoniae in Brooklyn, New York: epidemiology and recommendations for detection. Antimicrob Agents Chemother. 2005;49(7):3018-20.

7. Woodford N, Tierno PM Jr, Young K, Tysall L, Palepou MF, Ward $E$, et al. Outbreak of Klebsiella pneumoniae producing a new carbapenem-hydrolyzing class A beta-lactamase, KPC-3, in a New York Medical Center. Antimicrob Agents Chemother. 2004;48(12):4793-9.

8. Samra Z, Ofir O, Lishtzinsky Y, Madar-Shapiro L, Bishara J. Outbreak of carbapenem-resistant Klebsiella pneumoniae producing KPC-3 in a tertiary medical centre in Israel. Int J Antimicrob Agents. 2007;30(6):525-9.

9. Navon-Venezia S, Leavitt A, Schwaber MJ, Rasheed JK, Srinivasan A, Patel JB, et al. First report on a hyperepidemic clone of KPC-3-producing Klebsiella pneumoniae in Israel genetically related to a strain causing outbreaks in the United States. Antimicrob Agents Chemother. 2009;53(2):818-20.

10. Giakoupi P, Papgiannitsis CC, Miriagou V, Pappa O, Polemis M, Tryfinopoulou K, et al. An update of the evolving epidemic ob blaKPC-2-carrying Klebsiella pneumoniae in Greece (20092010). J Antimicrob Chemother. 2011;66(7):1510-3.

11. Giakoupi P, Maltezou H, Polemis M, Pappa O, Saroglou G, Vatopoulos A, et al. KPC-2-producing Klebsiella pneumoniae infections in Greek hospital are mainly due to a hyperendemic clone. Euro Surveill 2009;14(21):pii=19218. Available from: http://www.eurosurveillance.org/ViewArticle. aspx?Articleld $=19218$

12. Kontopoulou K, Protonotariou E, Vasilakos K, Kriti M, Koteli A, Antoniadou E, et al. Hospital outbreak caused by Klebsiella pneumoniae producing KPC-2-beta-lactamase resistant to colistin. J Hosp Infect. 2010;76(1):70-3.

13. Maltezou HC, Giakkoupi P, Maragos A, Bolikas M, Raftopoulos V, Papahatzaki H, et al. Outbreak of infections due to KPC2-producing Klebsiella pneumoniae in a hospital in Crete (Greece). J Infect. 2009;58(3):213-9.

14. Pournaras S, Protonotariou E, Voulgari E, Kristo I, Dimitroulia E, Vitti D, et al. Clonal spread of KPC-2 carbapenemase producing Klebsiella pneumoniae strains in Greece. J Antimicrob Chemother. 2009;64(2):348-52.

15. Souli M, Galani I, Antoniadou A, Papadomichelakis E, Poulakou $G$, Panagea T, et al. An outbreak of infection due to betaLactamase Klebsiella pneumoniae Carbapenemase 2-producing K. pneumoniae in a Greek University Hospital: molecular characterization, epidemiology, and outcomes. Clin Infect Dis. 2010;50(3):364-73.

16. Gaibani P, Ambretti S, Berlingeri A, Gelsomino F, Bielli A Landini MP, et al. Rapid increase of carbapenemase-producing Klebsiella pneumoniae strains in a large Italian hospital: surveillance period 1 March - 30 September 2010. Euro Surveill. 2011;16(8):pii=19800. Available from: http://www. eurosurveillance.org/ViewArticle.aspx?Articleld=19800

17. Carbonne A, Thiolet JM, Fournier S, Fortineau N, KassisChikhani N, Boytchev I, et al. Control of a multi-hospital outbreak of KPC-producing Klebsiella pneumoniae type 2 in France, September to October 2009. Euro Surveill. 2010;15(48): pii=19734. Available from: http://www. eurosurveillance.org/ViewArticle.aspx?Articleld=19734

18. Centers for Disease Control and Prevention (CDC). Guidance for control of infections with carbapenem-resistant or carbapenemase-producing Enterobacteriaceae in acute care facilities. MMWR Morb Mortal Wkly Rep. 2009;58(10):526-60.

19. Landman D, Salvani JK, Bratu S, Quale J. Evaluation of techniques for detection of carbapenem-resistant Klebsiella pneumoniae in stool surveillance cultures. J Clin Microbiol. 2005;43(11):5639-41.

20. Curiao T, Morosini MI, Ruiz-Garbajosa P, Robustillo A, Baquero F, Coque TM, et al. Emergence of bla KPC-3-Tn4401a associated with a pKPN3/4-like plasmid within $\mathrm{ST}_{3} 84$ and ST388 Klebsiella pneumoniae clones in Spain. J Antimicrob Chemother. 2010;65(8):1608-14 
21. Gregory CJ, Llata E, Stine N, Gould C, Santiago LM, Vazquez GJ, et al. Outbreak of carbapenem-resistant Klebsiella pneumoniae in Puerto Rico associated with a novel carbapenemase variant. Infect Control Hosp Epidemiol. 2010;31(5):476-84.

22. Lopez JA, Correa A, Navon-Venezia S, Correa AL, Torres JA, Briceno DF et al. Intercontinental spread from Israel to Colombia of a KPC-3-producing Klebsiella pneumoniae strain. Clin Microbiol Infect. 2010;17(1):52-6.

23. Wendt C, Schutt S, Dalpke AH, Konrad M, Mieth M, TrierweilerHauke B, et al. First outbreak of Klebsiella pneumoniae carbapenemase (KPC)-producing K. pneumoniae in Germany. Eur J Clin Microbiol Infect Dis. 2010;29(5):563-70.

24. Gasink LB, Edelstein PH, Lautenbach E, Synnestvedt M, Fishman NO. Risk factors and clinical impact of Klebsiella pneumoniae carbapenemase-producing K. pneumoniae. Infect Control Hosp Epidemiol. 2009;30(12):1180-5.

25. Hussein K, Sprecher H, Mashiach T, Oren I, Kassis I, Finkelstein R. Carbapenem resistance among Klebsiella pneumoniae isolates: risk factors, molecular characteristics, and susceptibility patterns. Infect Control Hosp Epidemiol. 2009;30(7):666-71.

26. Schwaber MJ, Klarfeld-Lidji S, Navon-Venezia S, Schwartz D, Leavitt A, Carmeli Y. Predictors of carbapenem-resistant Klebsiella pneumoniae acquisition among hospitalized adults and effect of acquisition on mortality. Antimicrob Agents Chemother. 2008;52(3):1028-33.

27. Nadkarni AS, Schliep T, Khan L, Zeana CB. Cluster of bloodstream infections caused by KPC-2 carbapenemaseproducing Klebsiella pneumoniae in Manhattan. Am J Infect Control. 2009;37(2):121-6.

28. Goldfarb D, Harvey SB, Jessamine K, Jessamine P, Toye B, Desjardins M. Detection of plasmid-mediated KPC-producing Klebsiella pneumoniae in Ottawa, Canada: evidence of intrahospital transmission. J Clin Microbiol. 2009;47(6):1920-2.

29. Larson EL, Cimiotti JP, Haas J, Nesin M, Allen A, Della-Latta P, et al. Gram-negative bacilli associated with catheterassociated and non-catheter-associated bloodstream infections and hand carriage by healthcare workers in neonatal intensive care units. Pediatr Crit Care Med. 2005;6(4):457-61.

30. Munoz-Price LS, Hayden MK, Lolans K, Won S, Calvert K,

Lin M, et al. Successful control of an outbreak of Klebsiella pneumoniae carbapenemase-producing K. pneumoniae at a long-term acute care hospital. Infect Control Hosp Epidemiol. 2010;31(4):341-7. 